\title{
High Order Calculation of the Multipole Content of Three Dimensional Electrostatic Geometries
}

\author{
Martin Berz, William M. Fawley and Kyoung Hahn \\ Lawrence Berikeley Laboratory, \\ University of Califomia \\ Berkeley, CA 94720
}

April 1991

* Work supported by the Director, Office of Energy Research, Office of Basic Energy Sciences, Advanced Energy Projects Division, U.S. Department of Energy, under Contract No. No. DE-ACO3-76SFO0098. 


\title{
High Order Calculation of the Multipole Content of Three Dimensional Electrostatic Geometries*
}

\author{
Martin Berz! William M. Fawley and Kyoung Hahn \\ Lawrence Berkeley Laboratory \\ University of Californis \\ Berkeley, CA 94720, USA
}

\begin{abstract}
We present an accurate and simple method of 3-D multipole decomposition of the field of arbitrary electrode geometries. The induced charge on the surface is obtained by inverting the capacity matrix. The multipole moment decomposition of the resulting potential is readily accomplished using Differential Algebra methodology. The method is applied to the focussing lattice geometry of the MIBE-4 accelerator at LBL. Multipole terms of up to the order 5 are computed and a numerical accuracy of $<1 \%$ is obtained. The effective quadrupole and dodecapole field strength are in good agreement with previous results.
\end{abstract}

\section{INTRODUCTION}

In many cases, the exact computation of higher order multipoles in electrostatic geometries is important. For example, in a real quadrupole-focussing geometry, multipole harmonics in addition to the wanted quadrupole one are always present to some degree. The strength of the multipole harmonics depends on the specific geometry of the electrodes, with sone harmonics being eliminated or ninimized by proper choice of electrode dimensions. The MAE-4, Multiple Beam Experiment at LBL, Jattice has many additional multipole components besides the wanted quadrupole component. Although weak, the presence of these components, in conjunction with non-negligible transverse beam displacements, will lead to emittance growth during beam transport[1][2]. To rectify this limitation, we computed the z-dependent strength of the multipole monents using a Differential Algebra (DA) methodol. ogy to decompose the thre-dimensional focussing potential. The latter is first determined by solving the capacity matrix of the actual electrode and aperture plate geometry. The choice of DA methodology was motivated by its ability to work to arbitrarily high order, with numerical accuracy limited only by that of the capacity matrix. This is in contrast to the more common method of determining the potential on is 3 -dimensional mesh, where both the unultipole order and numerical accuracy are limited by the mesil resolution. Our calculations show good agreement

-Work supported by the Ditectar, Olfice of Etuergy Resensch, OC. lire of Bwic Energy Sciencen, Advanced Energy Projects Division, U.S. Dept. of Energy, under Contract No. DE-AC03-76SFo0098.

iPresently at Michiga، Stale University with previous determinations of the effective quadrupole lengths and dodecapole strengths and we believe that this method may prove useful wherever multipole decomposition of complicated siatic fields is needed.

\section{POTENTIAL PROBLEM SOLUTION BY THE CAPACITY MATRIX METHOD}

When a complicated boundary shape is present, a conventional field solver using FFT or SOR techniques becomes computationally expensive because of the large number of mesh points required, especially in 3-D calculations. This difficulty can be alleviated noticeably by calculating the induced charge at the boundary surface directly rather than specifying the field boundary condition. Once the boundary charge distribution is known, the field at the location of a particle can be calculated straightforwardly. Since the number of required mesh nodes is confined to where the actual charges are, i.e. at the boundary, substantial reduction in total mesh points is obtained.

In the capacity matrix method, the electrodes are covered with test points $x_{i}$ (hereafter called "nodes"). Charge $Q_{i}$ located at the $x_{i}$ are determined such that the potential induced on $x_{i}$ by all the other nodes assumes the desired value. This reduces to solving the linear system

$$
\phi_{j}=\sum_{i} G\left(\mathbf{x}_{i}, \mathbf{x}_{j}\right) Q_{i}
$$

where $G\left(x_{i}, x_{j}\right)$ is the Green's function describing the cffect of the potential of a charge at $x_{i}$ to the point $x_{j}$. The inverse to $G\left(x_{i}, x_{j}\right)$ is often called the capacity matrix $\left(C_{i j}\right)$. Once the charge $Q_{i}$ are known, the potential at any point in space can be computed as

$$
\phi(x)=\sum_{i} G\left(\mathbf{x}_{i}, \mathbf{x}\right) Q_{1} .
$$

For non-overlapping test charges, one may use the simple Green's function of

$$
G\left(\mathbf{x}_{i}, \mathbf{x}_{j}\right)=\frac{1}{\left|x_{i}-x_{j}\right|} \quad \text { for } \quad i \neq j .
$$

When computing the self-potential $(i=j)$ or when the test charge width $\sigma_{i}$ exceeds an internode spacing, the test 
charge profile must be carefully considered. For the 3 D calculations discussed in Sec. 4, a triangular charge distribution was used with

$$
p(x)=\left\{\begin{array}{cl}
\frac{3}{\pi \sigma_{i}}\left(1-\frac{r_{i}}{\sigma_{i}}\right) & \text { if } r_{i} \leq \sigma_{i} \\
0 & \text { otherwise }
\end{array}\right.
$$

where $r_{i}=\left|x-x_{i}\right|$. With this profile, the potential within $\sigma_{i}$ of the teat charge is given by

$$
\phi(x)=\frac{Q_{i}}{\sigma_{i}}\left[2-2\left(\frac{r_{i}}{\sigma_{i}}\right)^{2}+\left(\frac{r_{i}}{\sigma_{i}}\right)^{3}\right]
$$

and

$$
C_{i i}^{-1}=\frac{2}{\sigma_{i}}
$$

Although the particular choice of the charge profile is somewhat arbitrary, however, numerical calculations show that the detirmination of the mutipole harmonics is not highly sensitive to the exact charge profile. The distribution width $\sigma_{i}$ is typically set to the internode spacing, depending on the charge distribution used. This choice prevents the self-potentia! from unphysically dominating the problem. Although we are representing the physically thin surface image charge by non-zero thickness spherical charges, the electric field on and near the beam transport axis should be little affected so long as the intornode spacing and charge distribution width $\sigma_{i}$ are small compared with the clear aperture between electrodes and the electrode-aperture plate separations in :

\section{MULTIPOLE EXPANSION AND DIFFERENTIAL ALGEBRA}

Once the boundary surface charge has been determined (e.g. by the capacity matrix method of Sec. 2), the electric potential at arbitrary point in space can be computed by the sunmation over the charges (see equation (2)). $\mathrm{Th}_{\text {; }}$ resulting approximate potential is infinitely differentiable, and thus its Taylor series can be computed. At interior positions where the potential varies smoothly, an approxinate functional expansion can prove extremely useful in turms of computational economics. Furthermore, the expansion is quite intuitive because certain nonlinearities of the transfer map couple only with certain multipole terms. For a system such as $\mathrm{MBE}-4$ where the quadrupole moment is by far the dominant component, an expansion around an aperture axis followed by a systematic multipole decomposition of the field is more convenient than the usual power serits expansion.

The multipole coefficients $M_{k, d}(z)$ of the potential $\phi$ are defined in cylindrical coordinates system by

$$
\phi(r, \theta, z)=\sum_{k=0}^{\infty} \sum_{l=0}^{\infty} h f_{k, 1}(z) r^{k} \cos (l \theta)
$$

where a Fourier series expansion in $\theta$ and power series in $r$ are used. Notice that the up-down symmetry, i.e. symmetric under $(y--y)$ transformation, of the MBE-4 ge- ometry is implicitly assumed. No z-axis expansion is performed and $M_{t, l}(z)$ is calculated at numerous locations in $z$.

The source-free ${ }^{1}$ vacuum potential $\phi$ satisfies the Laplace equation $\left(\nabla^{2} \phi=0\right)$ and thus the $M_{k, 1}$ observe the following recursion relation:

$$
M_{k, 1}=M_{k-2, l}^{\prime \prime} /\left(l^{2}-k^{2}\right),
$$

where double prime denotes the second derivative with respect to $z$. In order that neither $\phi$ nor $\nabla^{2} \phi$ be singular at $r=0$ and that $\phi$ be bounded at $z= \pm \infty$, the relation $k \geq l \geq 0$ and $k-l=$ even must be true for non-zero coefficients. The entire ensemble of multipole coefficients can then be determined from $M_{k, k}$ and its $z$-derivatives.

An explicit form for $M_{k, k}$ may be obtained by expressing $\phi$ as a Taylor series, i.e.

$$
\phi(x, y, z)=\sum_{m} \sum_{n} \frac{x^{m} y^{n}}{m ! n !}\left(\frac{\partial}{\partial z}\right)^{m}\left(\frac{\partial}{\partial y}\right)^{n} \phi(0,0, z)
$$

After equating this expression with that of eqn. (7) and integrating with $\cos (k \theta)$, one finds for $k \geq 1$

$$
M_{k, k}(z)=\sum_{n=0, \text { even }}^{k} \frac{(-1)^{(n / 2)}}{2^{k-1} n !(k-n) !}\left(\frac{\partial}{\partial x}\right)^{(k-n)}\left(\frac{\partial}{\partial y}\right)^{n} \phi
$$

where the up-and-down symmetry has again been used and $\phi$ is given by the summation over the induced boundary charges,

$$
\phi(x)=\sum_{i=1}^{N} \frac{Q_{i}}{r_{i}}
$$

Here $r_{i} \equiv\left|x-x_{i}\right|$ and $Q_{i}$ is the charge at the position of the $i^{\text {th }}$ node. Away from the nodes this function is infinitely differentiable, and it is in principle possible (although very tedious for high orders) to compute the required derivatives $M_{k, k}^{(2 i)}$ by differentiating expression (10).

To circumvent this difficulty, we use differential algebraic techniques for the computation of the higher order derivatives to machine precision without numerical errors. These techniques, which also play a key role in the high order description of optical systems, have been discussed in detail elsewhere[3][4], and the interested reader is referred to the above mentioned papers.

\section{COMPUTATIONAl RESULTS}

A FORTRAN code was written to evaluate the $G\left(x_{i}, \mathbf{x}_{j}\right)$ of any electrode geometry, and then invert them to obtain the capacity matrix $C_{i j}$ and the node charges $Q_{i}$.

In the case of the actual MBE-4 focussing lattice, there are a number of separate elements in the electrode: a)

\footnotetext{
TWhen beam apace charge is present, it is convenient to decompose the total field as a sum of vacuum potential from the boundary charges and the free space potential of the beam charge. Hence, the relevant potential in equation (1) is the difference between the spec. ified boundary potential and that due to the beam space charge in the abence of boundaries.
} 
Quadrupole electrodes which are held at either ground or a negative voltage, b) Flat aperture plates from which the electrodes are cantilevered and through whose holes the four ion beams t ravel, c) A large "can" enclosure surrounding the entire focussing lattice.

The nodes were distributed uniformly over the surface of the electrode rods and with an $\sim 1 / r$ dependence on the endplates. The charge distribution width $\sigma_{i}$ was set to a constant ( $\approx 2.5 \mathrm{~mm}$ for tutal node number $N=5000$ ), somewhat smaller than the typical internode distance in order to minimize the size effect.

Since our main interest was calculation of threedimensional multipole components of general potential geometries, the potential at the electrostatic quadrupole electrodes was set to $\pm V_{q}$ rather than grounding one of the electrode pairs as in the actual case for MBE-4. We have also neglected the "can" enclosure.

In order to evaluate expression (10) numerically, higher order differential algebras were used for the computation of the derivatives of the potential $\phi$. From there, the $M_{k, d}$ were computed from $0^{t h}$ to $6^{t h}$ order computed using expression (8).

The coefficients were made dimensionless by scaling the potential by a factor of $V_{q}$ and using a normalization length of the aperture radius $a$, i.e.:

$$
\phi=V_{q} \sum_{k=0}^{6} \sum_{l=0}^{6} M_{k, l}(z)\left(\frac{r}{a}\right)^{k} \cos (l \theta) .
$$

Figure 1 shows the results for the case of $N=5000$. Our inidplane multipole moments of $M_{2,2}=0.9855$ and $M_{6,6}=$ 0.03460 are in good agreement with 2 -dimensional (i.e. $z$ independent) values of 0.9658 and 0.03461 respectively by Brady [5] using POISSON program. Meuth et al. [6] experimentally determined an effective quadrupole length of $10.11 \pm 0.14 \mathrm{~cm}$ by measuring the phase advance of the centroid motion per lattice period $\left(\sigma_{0}\right), 5 \%$ shorter than our predicted effective length ( $\equiv \int M_{2,2}(z) d z / M_{2,2}(z=0)$ ) of $10.60 \mathrm{~cm}$ (one should note that their most reliable measurement at $\sigma_{o}=72^{\circ}$ gave an effective quadrupole length of $9.99 \mathrm{~cm}$ ). Our calcuiation, however, is quite close to the actual electrode length of $10.74 \mathrm{~cm}$.

Near the endplates, the ficld contains large multipole field components because of the inter-digitai structure of the yuadrupole rods, their closeness to the endplates, and the beam aperture holes. Our numerical errors may be larger in this rcgion because $\sigma_{i}$ exceeds the optimal value around the aperture holes and the spherical charge shape might be a poor approximation to the true image charge distribution on the flat endplates. Thus, perhaps additional nodes and a variable charge distribution width and slıape would be required in order to maintain $1 \%$ accuracy everywhere.

\section{CONCLUSION AND DISCUSSION}

We have presented a simple and accurate method of determining the multipole field components of electrostatic forcussing system with arbitrary geometry. First the in. duced surface charge distribution for a given electrode potential is obtained by inverting the eapacity matrix, followed by multipole decomposition of the field at the beam axis by using the DA techniques. Since the charge on the conductor surface is calculated directly in this method, the multipole harmonic amplitude can be obtained by the summation of the contributions from all the charges. Although finite size of the charge distribution at a given node poses some uncertainty of the determination of the capacity matrix, the effect can be minimized by optimally choosing the profile shape and width. Numerical experiments show that the decomposition is indeed insensitive to the the charge distribution at the nodes so long as the internode distance is much smaller than the aperture radius.

For the actual MBE-4 accelerator lattice structure, the calculated, $z$-dependent amplitudes of the multipole harmonics show good agreement with previous measurements in terms of effective quadrupole length and dodecapole strength.

\section{References}

[1] C. Celata, I. Haber, L. Laslett, L. Smith and M. Tiefenback, IEEE Trans. on Nucl. Sci. NS-32 (1985) 2480.

[2] S. Eylon, E. Colby, T. Fessenden, T. Garvey, K. Hahn and E. Henestroza, Part. Acc. to be published.

[3] M. Berz, IEEE Trans. Elec. Dev., 35-11 (1988) 2002.

[4] M. Berz, Part. Acc., 24 (1989) 109.

[5] V. Brady, private communication (1987).

[6] H. Meuth, S. Eylon, E. Henestroza and K. Hahn, private communication (1989).

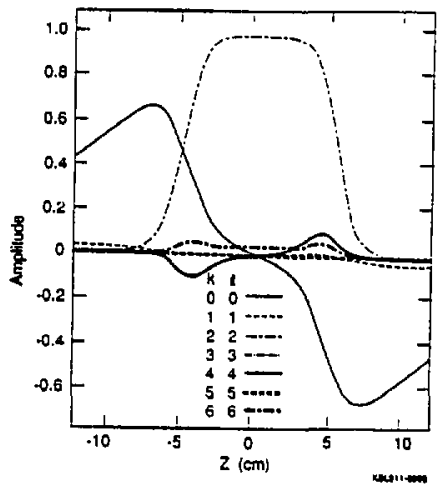

Fig. 1 Multipole decomposition of the MBE-4 lattice plotted versus $z$ for "pure" multipoles $M_{k, k}$. 